\title{
Using advanced X-ray and neutron diffraction techniques in single molecule magnets research
}

\author{
J. Overgaard, E. Damgaard-Møller, L. Krause, I. Kibalin, E. A. Klahn \\ Department of Chemistry, Aarhus University, Langelandsgade 140, DK-8000 Aarhus C, Denmark, ${ }^{2} L L B$, CEA, CE de Saclay, Gif sur \\ Yvette, 91191, France \\ jacobo@chem.au.dk
}

Single-molecule magnet (SMM) is the generic name given to a broad class of molecules, which exhibit an energy barrier to magnetization reversal. In simpler terms, SMMs have that special trait that once they have become magnetized by an external magnetic field, the induced magnetic moment (which we, for simplicity, could call spin up or spin down) resists reorientation. For that reason, such fascinating molecules are envisaged to act as molecular bits, or quantum bits, qubits. The origin of this effect is magnetic anisotropy, i.e. the different magnetic response to an external field (quantified by the magnetic susceptibility) depending on the relative orientation of field and molecule. Magnetic anisotropy splits the magnetic substates, and the reason for this is the presence of unquenched orbital angular momentum. Thus, at the very core, to be able to develop novel SMMs we need to understand how to control the electronic ground state of a complex. This has followed two paths, depending on whether the electron-carrier is a $3 \mathrm{~d}$ or $4 \mathrm{f}$ element.

For 4f-based SMMs, a widespread approach has aimed at developing complementary ligand fields relative to the valence electron density shape of the most magnetic Mj-state of the 4f-ion in question. However simple and unvalidated by experiment, this approach has been fantastically useful. Recently, we showed how the experimental electron density from X-ray diffraction could reveal hitherto unseen details in the electronic structure of a Dy-based SMM, thus elucidating the mechanism[1]. For 3d-systems, the ligand field is much stronger and the approach is thus different. The magnetic anisotropy is enhanced in distorted tetrahedral complexes of Co ${ }^{\mathrm{II}}$, as has recently been shown[2-4].

Herein, I will show how a combination of high-resolution synchrotron X-ray diffraction (XRD) and polarized neutron diffraction (PND) can be used to quantify the magnetic anisotropy in $\left[\mathrm{CoX}_{2} \mathrm{tmtu}_{2}\right](\mathrm{X}=\mathrm{Cl}, \mathrm{Br}$, tmtu $=$ tetramethylthiourea). The $\mathrm{XRD}$ data provides a multipole model of the electron density, while the PND provides the full magnetic susceptibility tensor. The experimental results are supported by ab initio calculations.

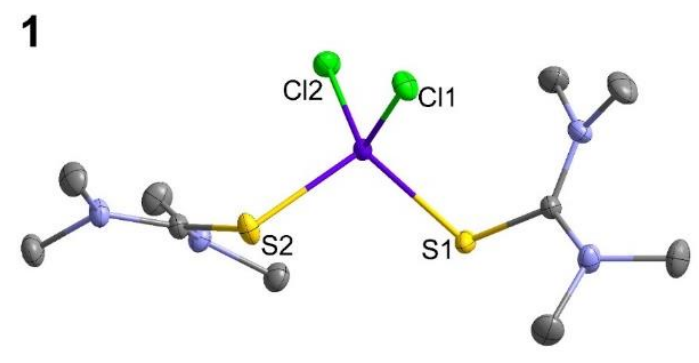

Figure 1. ORTEP drawing of the Cl-complex studied here based on $20 \mathrm{~K}$ synchrotron data, showing $90 \%$ ellipsoids.

[1] Gao, C., Genoni, A., Gao, S., Jiang, S., Soncini, A. \& Overgaard, J. (2020). Nat. Chem. 12, 213.

[2] Vaidya, S., Shukla, P., Tripathi, S., Rivière, E., Mallah, T., Rajaraman, G. \& Shanmugam, M. (2018). Inorg. Chem. 57, 3371.

[3] Rechkemmer, Y., Breitgoff, F. D., van der Meer, M., Atanasov, M., Hakl, M., Orlita, M., Neugebauer, P., Neese, F., Sarkar, B. \& van Slageren, J. (2016). Nat. Commun. 7, 10467.

[4] Damgaard-Møller, E., Krause, L., Tolborg, K., Macetti, G., Genoni, A. \& Overgaard, J. (2020). Angew. Chem. Int. Ed. 59, 21203.

Keywords: Single molecule magnets, electron density, polarized neutron diffraction, synchrotron, multipole model 